\title{
Effects of tai chi on cognition and instrumental activities of daily living in community dwelling older people with mild cognitive impairment
}

Mei-yi $\operatorname{Siu}^{1 *}$ and Diana T. F. Lee ${ }^{2}$

\begin{abstract}
Background: Cognitive impairment places older adults at high risk of functional disability in their daily-life activities, and thus affecting their quality of life. This study aimed to examine the effects of Tai Chi on general cognitive functions and instrumental activities of daily living (IADL) in community-dwelling older people with mild cognitive impairment (MCl) in Hong Kong.

Methods: The study adopted a multi-site nonequivalent control-group pretest-posttest design. 160 communitydwelling older people, aged $\geq 60$, with $\mathrm{MCl}$, from four community elderly centers participated in the study. The intervention group $(I G, n=80)$ received training in the Yang-style simple form of Tai Chi, at a frequency of two lessons per week for 16 weeks. Each lesson lasted for one hour. The control group (CG, $n=80)$ had no treatment regime and joined different recreational activity groups in community centers as usual within the study period. Outcome measures included measures of global cognitive status and IADL. The Chinese version of the Mini-Mental State Examination (CMMSE) was used for global cognitive assessment. The Hong Kong Chinese version of Lawton's Instrumental Activities of Daily Living (IADL-CV) was used to assess the participants' IADL levels. General Estimating Equations (GEE) was used to examine each of the outcome variables for the two groups at the two study time points (the baseline and at the end of the study). Meanwhile, minimum detectable change (MDC) was calculated to estimate the magnitude of changes required to eradicate the possibility of measurement error of outcome measures.
\end{abstract}

Results: Seventy four participants in the IG and 71 participants in the CG completed the study. With adjustments for differences in age, education, marital status and living conditions, the findings revealed that the participants in the IG scored significantly better on the CMMSE test $(P=0.001)$, and the instrumental ADL questionnaire $(P=0.004)$. However, those scores changes did not exceed the limits of the respective MDCs in the study, the possibility of measurement variation due to error could not be excluded.

Conclusion: Tai Chi may be an effective strategy to enhance cognitive health and maintain functional abilities in instrumental ADL in older people with $\mathrm{MCl}$.

Trial registration: NCT03404765 (Retrospectively registered January 19, 2018)

Keywords: Tai chi, Cognition, Instrumental activities of daily living, Mild cognitive impairment

\footnotetext{
* Correspondence: minniesiu@twc.edu.hk

${ }^{1}$ The School of Nursing, Tung Wah College, 31 Wylie Road, Homantin,

Kowloon, Hong Kong, SAR, People's Republic of China

Full list of author information is available at the end of the article
} 


\section{Background}

As ageing population is rising all over the world, agerelated cognitive decline has been highlighted as a public health concern worldwide. Nowadays, 47.5 million people worldwide live with dementia, and there are 7.7 million new cases every year. These figures are projected double every twenty years, reaching 75.6 million in 2030 and 135.5 million in 2050 [1]. In the Asia Pacific region, people diagnosed with dementia are expected to increase from 27 million in 2015 to 70 million by 2050 [2].

The aging population in Hong Kong is largely a consequence of a longer life expectancy and lowering fertility rate. According to the Census and Statistic Department (2015), the proportion of elderly citizens aged 65 or above reached $15 \%$ in 2014 and is projected to rise markedly to $33 \%$ in 2064 [3]. Thus, dementia occurred in $9.3 \%$ of people aged 70 and above in 2008 and is expected double for every 5 years of increase in age [4]. The prevalence rate of dementia is projected to increase by $222 \%$ from 2009 to 2039 [5].

With increasing awareness about the importance of promoting cognitive health in the aging population, mild cognitive impairment (MCI) has become a major research topic in the field of dementia and related cognitive disorders. MCI has been described as an intermediate stage of cognitive deterioration, from normal cognition to fully developed symptoms of dementia [6]. Persons with MCI pose with greater risk of developing dementia [7].

\section{Cognitive and functional deficits in mild cognitive impairment}

Cognitive and functional impairments are hallmark features to distinguish the difference between $\mathrm{MCI}$ and dementia. Although the general cognitive functions and basic activities of daily living (BADL) such as bathing, continence, dressing, feeding, toileting and transferring, are largely preserved, numerous researchers have suggested that people with MCI may experience some deficits in performing instrumental activities of daily living (IADL), including the ability on using telephone, shopping, preparing food, doing house chores and laundry, using transportation, self-medication, and managing financial affairs etc., comparing to age-matched cognitively healthy controls $[8,9]$. IADL enactment must draw multiple cognitive domains for competent performance. Subtle difficulties illustrated by slowness and hesitation in carrying out complex ADL tasks may indicate some degree of dysfunction [10-14]. Highly cognitively demanding IADL was found to be positively correlated with performance in six cognitive domains, namely memory, attention, processing speed, executive function, language, and visuospatial ability [13]. Thus, MCI plus IADL limitations are associated with higher conversion and shorter duration to clinical manifestation of dementia [15-17].

While people with MCI have high chances of becoming demented, the heterogeneous nature of $\mathrm{MCI}$ may carry an unstable prognosis. MCI is not necessarily a prodromal syndrome of dementia, as many people not showing deterioration but become static in the status of their cognitive deficits, and in some cases, may revert to normal cognition $[18,19]$. Persons with MCI may benefit from early interventions by slowing down the rate of progression to dementia.

\section{Tai chi as a strategy against cognitive and functional declines}

At present, there is no consensus among medical scientists on any kind of drug treatment for people with MCI. Clinical trials of drugs used for the treatment of Alzheimer's disease have shown no beneficial effects on delaying progression from MCI to dementia [20-22]. Furthermore, these cognitive-enhancing drugs can cause gastro-intestinal disturbances [22].

Research on non-pharmacological intervention, however, has suggested that lifestyle factors can help prevent dementia. Fratiglioni, Paillard-Borg, and Winblad (2004) identified three lifestyle factors that may contribute to slowing down cognitive decline, which included integrated social networking, cognitively stimulating activity, and regular physical activity [23]. An epidemiological study of elderly persons in Britain, with 21 years' follow up, observed that both cognitive and physical activity had protective effects against cognitive decline [24]. Another retrospective cohort study in Dijon and Montpellier (France), analyzed lifestyle data from 5698 non-demented older citizens from 1999 to 2001. The result also suggested that cognitively stimulating leisure activities might delay the onset of dementia in community-dwelling older people [25].

Mind-body exercise, such as Tai Chi, is a type of physical exercise that combines physical and cognitivestimulating activity. Tai Chi focuses on tranquility of mind to achieve longevity through meditation and life style modification [26]. It is an alternate form of aerobic exercise with moderate intensity [27]. Aerobic exercise can delay age-related brain atrophy, increase cerebral blood circulation, and stimulate neural cell regeneration $[28,29]$. As an activity to promote cognitive stimulation, the meditation component of Tai Chi can improve attentional focus and executive function through learning a series of choreographed movements in continual sequence [30, 31]. As well, engaging in Tai Chi exercise in group settings allows elderly persons to maintain social contact with others, which can further benefit cognition through stress reduction and peer group support [32, 33]. 
Systematic reviews and meta-analysis studies have reported Tai Chi training can improve general cognitive performance, memory, attention, language, and executive functions in older adults, including people with MCI [34-37]. Effective management of IADL is dependent on memory, attention, and executive functions. [12, 13, 38]. Tai Chi can be conceived as an appropriate exercise for elderly persons to preserve cognitive health and functional abilities.

As aging populations increase rapidly all over the world, interventions to maintain cognitive functioning in elderly people will be of paramount important to preserve functional ability, independence, and HRQOL [34]. Cognitive impairment is one of the strong determinants of functional disability $[39,40]$. Currently, there is limited research evidence to support the effects of Tai Chi training on IADL performance. Therefore, the aim of this study is to examine the effects of Tai Chi on general cognitive functions and instrumental activities of daily living (IADL) in community-dwelling older people with mild cognitive impairment (MCI) in Hong Kong.

\section{Methods}

\section{Study design}

This was a quasi-experimental study which adopted a multisite nonequivalent control group pre- and post-test design (Clinical trial registry: CREC Ref. No.:2015.348).

\section{Participants and settings}

Convenience sampling was used to recruit participants from four community elderly centers. These elderly centers are run by non-government organizations which are registered under and monitored by the Social Welfare Department of Hong Kong. There are around 50 community elderly centers in Hong Kong. To gain access to the target population for this study, various elderly centers in different regions in Hong Kong were contacted. Each elderly center in the study has an enrollment of 200 to 300 members. They all operate with similar settings. Two of these centers were randomized as intervention groups, and the other two were treated as control groups. Recruitment process started after center assignment, and the participants were allocated to the experimental or control groups according to the centers to which they belonged. This arrangement avoided possible contamination of the experiment by diffusing the Tai Chi learning.

The domains of cognitive defect experienced by individuals with MCI vary. As the sample population in the study might comprise of different subtypes of $\mathrm{MCI}$ persons, the general cognitive function, as measured by the Chinese-Mini Mental State Examination (CMMSE) [41], was chosen as a screening criterion and an outcome variable for this study.
The inclusion criteria for the study were: (1) Chinese people aged 60 years or above; (2) the CMMSE screening score ranging from 19 to 28 , which was corrected based on educational level ( $\geq 18$ for illiterate respondents and $\geq 22$ for those having received more than two years of schooling) [42]. The CMMSE score of 19 was set as the bottom limit because previous studies have found that older adults with MMSE scores of 18 can provide valid answers to inquiries and are able to follow instructions [43, 44]. At the upper limit, some elderly persons with MMSE scores of 28 may exhibit symptoms of memory deficit [45]; (3) ability to perform self-care functions on their own; (4) no confirmed diagnosis of dementia, depression or other psychiatric illnesses which would interfere with cognitive performance; and (5) no engagement in any structured physical exercise program or Tai Chi practice in the preceding year.

Elderly persons were excluded from the study if they: (1) had a medical history of chronic alcoholism or brain trauma occurred in previous years that could interfere with cognition; (2) had been regular users of medications that could affect cognition; or (3) had clinical conditions that contraindicated light to moderate physical exercise.

The participants were requested not to join any structured exercise training other than the Tai Chi interventions during the study period.

\section{Sample size estimation}

A medium effect size of 0.5 was set for the study, based on the previous research on the effect of Tai Chi on cognitive function [46]. With the use of a sample size calculator (https://www2.ccrb.cuhk.edu.hk/stat/Other.htm), a sample of 64 per group was expected to provide $80 \%$ power to detect a medium effect size of 0.5 , at a significance level of 0.05 [47]. Allowing for an attrition rate of $20 \%$ in the Tai Chi groups $[46,48,49], 77$ participants of each group would be needed to maintain sufficient power in the current study. To round up, 80 participants were recruited for each group.

\section{Intervention protocol Intervention group}

The intervention group (IG) received a 16-week Tai Chi program, of 32 sessions ( 2 sessions per week), each being one-hour long. The capacity was around 20 people per class. A certified Tai Chi master taught the Yang-style simple form of Tai Chi to the participants allocated to the IG. This Yang-style form was designed by a group of Tai Chi experts from the Chinese Sports Commission in 1956, and based on the Yang Tai Chi long form. It is sometimes called the Beijing or Peking form of Tai Chi, or the Tai Chi 24 form, because it consists of 24 unique movements [50]. There were four sets of movements included in the simplified form, that lesser time was 
required for the elderly practitioners, who usually had less exercise endurance, to complete the whole set of movements. Moreover, it was an appropriate form of Tai Chi for elderly persons with MCI because it involved only a few postures, and hence it was easier for them to learn and remember (Additional file 1: Table S1). The Tai Chi classes were conducted in the open hall of the community center or a public playground with sufficient space, a flat floor and good lighting.

Each Tai Chi session had three parts. The first part was the "warm up", which was comprised of simple motions to help the elderly learners to relax their muscles and joints. The second part was "Tai Chi instruction". The Tai Chi instructor taught the whole set of unique movements of the Yang-style simple form of Tai Chi to the groups, instructed them to move in circular motions with low speed and explained how to focus on breathing and muscle coordination. The final part was the "cool down", which involved activities to cease the Tai Chi and rest the body.

To ensure adherence, the participants were required to sign attendance records. Telephone follow-up was arranged to provide emotional support, reinforcement and compliance if they were absent from two consecutive lessons. As well, the participants were advised to do daily practice at home to foster better mastering of the Tai Chi movements and build up the regular exercise habit. A guide book was issued to each participant, providing guidance to reinforce correct postures. As it was difficult to ensure the accuracy of participants' selfreporting of their home practices, the out-of-class practice records served only as a source of communication to reinforce compliance with the Tai Chi practice.

\section{Control group}

The control group (CG) was treated as the usual care group and no exercise training was arranged. They were advised to attend different kinds of recreational activities provided by their elderly centers and to continue their daily activities, including their usual general physical mobility and social activities during the study period.

\section{Outcome measures}

\section{The Chinese version of mini-mental state examination (CMMSE)}

The CMMSE is a global cognitive assessment tool which has been translated from the original MMSE version developed by Folstein, Folstein, and McHugh, in 1975 [51]. The CMMSE is divided into two parts. The first part of the instrument assesses respondent's abilities in orientation, memory, and attention. The second part is to test respondent's abilities in naming objects, understanding verbal and written commands, repeating short sentences, and drawing overlapping polygons [41].
The sensitivity and the specificity of cut-off score of the CMMSE at $19 / 20$ were $97.5 \%$ and $97.3 \%$ respectively. The test-retest reliability of the instrument was 0.78 and the inter-rater reliability was 0.99 [41]. The CMMSE score ranges from 0 to 30 ; the higher the scores, the better cognitive performance implies.

\section{The Hong Kong Chinese version of Lawton's instrumental activities of daily living (IADL-CV)}

The Hong Kong Chinese version of Lawton's Instrumental Activities of Daily Living (IADL-CV) [52] is translated from the original English version of Lawton's IADL scale [53]. Nine domains of IADL are assessed, including ability of using telephone, shopping, preparing food, doing house-keeping and laundry tasks, using transportation, managing finances, handling medication, and doing handyman work. The inter-rater and the testretest reliabilities of the scale were 0.99 and 0.90 respectively. The Cronbach alpha assessing internal consistency was 0.86 . The higher the scores of each domain, the better the functional abilities of the elderly persons to live independently in the community represent [52].

\section{Data collection process}

All participants, both in the CG and the IG, received pre-study and post-study assessments based on identical sets of questionnaires. Demographic data and the baseline measurements for both groups were collected at the beginning of the study (T0). Post-study assessment (T1) was done 1-2 weeks after completion of the Tai Chi intervention.

\section{Data analysis}

The IBM SPSS Version 24.0 (IBM Crop. Armonk, N.Y.) was used for data entry and analysis. All statistical tests involved were 2-tailed and the level of significance was set at 5\%. Descriptive statistics, including mean, standard deviation, frequency, and percentage, were used to summarize and present the sample characteristics and outcome measures.

Chi-square, Fisher's exact, and independent $t$ - tests were used, as appropriate, to compare the characteristics of IG and CG at the baseline. If there were significant differences between two groups of these characteristics, they were adjusted in the subsequent outcome analysis. Standard error of measurement (SEM) and minimum detectable change (MDC) were calculated to estimate the magnitude of changes needed to confidently rule out the possibility of change in outcome measures owing to measurement error.

Generalized Estimating Equations (GEE) was applied for comparing each of the repeated measures study outcomes (i.e. CMMSE score, IADL-CV score) between the two groups with adjustment for those demographic 
characteristics showing statistical incomparability between groups. Two dummy variables, Group and Time, were included in each GEE model to represent the baseline group difference (Intervention - Control) and the time effect on the control group (Post 16 weeks - Baseline), respectively. Furthermore, the interaction term, Group by Time (Group*Time) was also included to assess the differential change in each outcome between the two groups. GEE model could account for intracorrelated repeated measures outcome and produce unbiased effect estimated under the data missingness mechanism of missing completely at random (MCAR).

\section{Ethical considerations}

Approval for conducting the study was obtained from the Joint CUHK-NTEC Clinical Research Ethics Committee of The Chinese University of Hong Kong and the selected community elderly centers.

The participants were required to sign a consent form. Information and the purpose of the study were explained. They could withdraw from the study at any time of their own will.

\section{Results}

The study was conducted from December 2015 to September 2016. A total of 178 elderly persons was screened for this study. Out of the 87 elderly persons (48.9\%) screened for the IG, seven (8.0\%) were rejected after the initial screening process. Of the 91 elderly persons (51.1\%) screened for the CG, 11 (21.1\%) were rejected. For the 18 rejected, 11 had scored very low marks in the CMMSE screening and the other seven were doing regular exercise. Eventually, there were 80 participants eligible for the IG and another 80 were admitted to the CG.

Throughout the study, there were six and nine participants dropped out for various reasons in the IG and CG respectively. The dropout rates were $7.5 \%$ for the IG and $11.25 \%$ for the CG (Fig. 1). The demographic characteristics of the completers and non-completers were basically comparable, and no significant difference was found.

The average attendance rate of all participants in the IG was $81.4 \%$. Reasons for not attending the Tai Chi classes were mainly due to attending medical follow-up, not feeling well or not being suitable to carry out outdoor exercise in poor weather conditions. There was no reason for missing classes due to any discomfort after practicing Tai Chi. No injury report was obtained during the Tai Chi intervention.

$73.8 \%$ female $(n=118)$ to $26.3 \%$ male $(n=42)$. A baseline demographic assessment showed that there was no significant difference between the two groups for gender

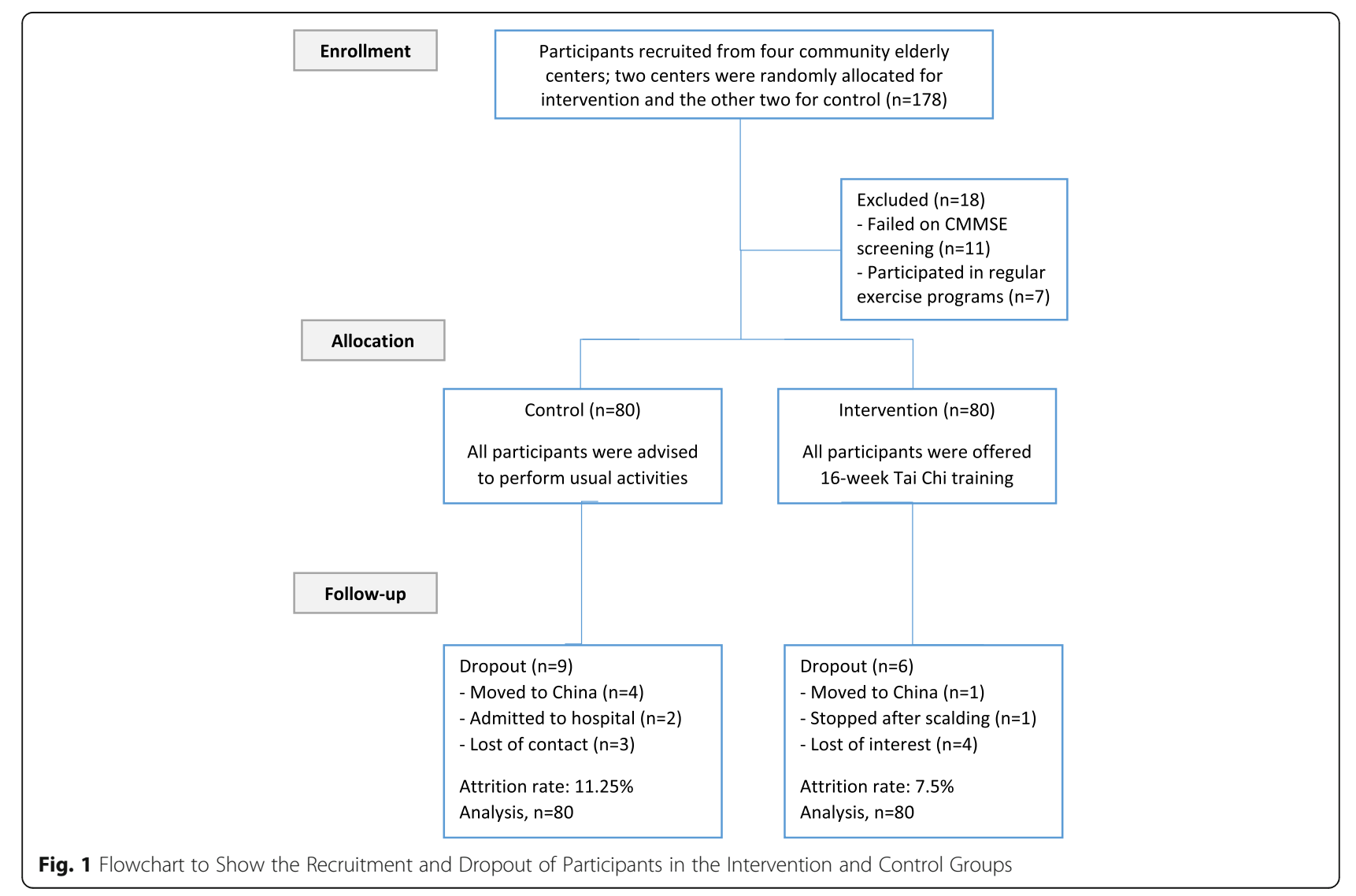


$(P=0.72)$ or comorbid conditions $(P=0.278)$. However, significant differences between groups were observed in some of the demographic variables, including age, education level, marital status and living conditions. The participants in the IG were younger $(P=0.001)$, with higher academic attainment $(P=0.004)$ than those in the CG. However, there were more participants in the CG than in the IG who lived alone $(P=0.013)$ and reported their status as widowed/single/divorced $(P=0.05)$ (Table 1 ).

The mean scores of CMMSE and IADL-CV for both CG and IG at the baseline and after 16 weeks of intervention, as well as their mean change scores are shown in Table 2. The IG showed greater improvement in both outcomes than the CG.

General Estimating Equations (GEE) were used to compare the differential changes between groups in the outcome variables after 16 weeks of intervention, with adjustments for the demographic variables which were shown to be statistically incomparable, including age, education level, living conditions and marital status. After adjusting the potential confounding factors, the IG showed significantly greater improvement in CMMSE score than the CG, over the 16-week study period (group by time interaction, $B=1.33,95 \%$ CI $0.53-2.13$, $P=0.001$; Cohen's $d$ effect size 0.50). Moreover, the IG revealed a significantly better improvement in IADL scores. The group by time interaction term was statistically significant $(B=1.07,95 \%$ CI $0.34-1.81, P=0.004$; Cohen's d effect size 0.48) (Table 3). Although participants of the IG demonstrated significantly greater improvements in both CMMSE and IADL-CV scores than the CG throughout the post-pre-study period, such changes in both groups were smaller than their MDCs and therefore failed to rule out the possibility of measurement variation due to error (Table 2).

\section{Discussion}

The key findings of current study suggested that elderly persons with MCI who practiced Tai Chi yielded significant improvement in general cognitive functions and IADL when compared to those participants with MCI without practicing Tai Chi, after adjusting the age difference, education level, marital status and living conditions for the two study groups, over the 16 weeks of the study period.

In the current study, CMMSE was used as baseline cognitive screening and subsequent cognitive assessment for all participants. There were several advantages for using CMMSE as a cognitive assessment tool. First, administration of the test took around 5 to $10 \mathrm{~min}$ for each participant, which was not overloaded for elderly participants. More importantly, MMSE has been used

Table 1 Baseline demographic characteristics of the participants

\begin{tabular}{|c|c|c|c|c|c|}
\hline Demographic characteristics & $\begin{array}{l}\text { Overall } \\
(n=160) \\
\text { Frequency (\%) }\end{array}$ & $\begin{array}{l}\text { Control } \\
(n=80) \\
\text { Frequency (\%) }\end{array}$ & $\begin{array}{l}\text { Intervention } \\
(n=80) \\
\text { Frequency (\%) }\end{array}$ & $\begin{array}{l}\text { Chi-Square } \\
\left(X^{2}\right)\end{array}$ & $P$-value \\
\hline Sex & & & & 0.13 & 0.719 \\
\hline - Male & $42(26.3)$ & $20(25)$ & $22(27.5)$ & & \\
\hline - Female & $118(73.8)$ & $60(75)$ & $58(72.5)$ & & \\
\hline Age & & & & 10.19 & $0.001^{*}$ \\
\hline$-60-74$ & $107(66.9)$ & $44(55.0)$ & $63(78.8)$ & & \\
\hline - 75 or above & $53(33.1)$ & $36(45.0)$ & $17(21.3)$ & & \\
\hline Education Level & & & & 8.23 & $0.004^{*}$ \\
\hline - Illiterate or below primary school & $70(43.8)$ & $44(55.0)$ & $26(32.5)$ & & \\
\hline - Completed primary school or above & $90(56.3)$ & $36(45.0)$ & $54(67.5)$ & & \\
\hline Marital Status & & & & 7.76 & $0.005^{*}$ \\
\hline - Married & $101(63.1)$ & $42(57.5)$ & $59(73.8)$ & & \\
\hline - Single/ Widowed/ Divorced & $59(36.9)$ & $38(47.5)$ & $21(26.3)$ & & \\
\hline Living Condition & & & & 6.18 & $0.013^{*}$ \\
\hline - Living alone & $35(21.9)$ & $24(30.0)$ & $11(13.8)$ & & \\
\hline - With family members & $125(78.1)$ & $56(70.0)$ & $69(86.3)$ & & \\
\hline Comorbidity & & & & & 0.278 \\
\hline-0 & $33(20.6)$ & $14(17.5)$ & 19 (23.8) & & \\
\hline$-1-2$ & $100(62.5)$ & $49(61.3)$ & $51(63.8)$ & & \\
\hline - 3 or more & 27 (16.9) & $17(21.3)$ & $10(12.5)$ & & \\
\hline
\end{tabular}


Table 2 Outcome variables across time between the study groups

\begin{tabular}{|c|c|c|c|c|c|c|}
\hline \multirow[t]{2}{*}{ Outcome variables } & \multicolumn{3}{|c|}{ Control $(n=80)$} & \multicolumn{3}{|c|}{ Intervention $(n=80)$} \\
\hline & Mean (SD) & SEM & $\begin{array}{l}\text { MDC } \\
(95 \% \mathrm{Cl}) \\
\end{array}$ & Mean (SD) & SEM & $\begin{array}{l}\text { MDC } \\
(95 \% \mathrm{Cl})\end{array}$ \\
\hline \multicolumn{7}{|l|}{ CMMSE } \\
\hline - Baseline & $24.61 \pm 2.75$ & 1.29 & 3.58 & $25.46 \pm 1.89$ & 0.89 & 2.46 \\
\hline - Post 16 weeks & $24.70 \pm 2.90$ & & & $26.74 \pm 2.42$ & & \\
\hline - Change of score & $0.11 \pm 2.78$ & & & $1.38 \pm 2.22$ & & \\
\hline \multicolumn{7}{|l|}{ IADL-CV } \\
\hline - Baseline & $24.20 \pm 2.55$ & 0.81 & 2.25 & $25.03 \pm 1.76$ & 0.56 & 1.56 \\
\hline - Post 16 weeks & $23.96 \pm 3.01$ & & & $25.88 \pm 1.65$ & & \\
\hline - Change of score & $-0.21 \pm 2.69$ & & & $0.84 \pm 1.82$ & & \\
\hline
\end{tabular}

Abbreviations: CMMSE Chinese version of Mini-Mental State Examination, IADL-CV Hong Kong Chinese version of Lawton Instrumental Activities of Daily Living scale

widely in clinical research to evaluate cognitive function and impairment. It facilitated comparisons and discussions of findings of the current study with previous cognitive research.

However, some researchers criticized that MMSE was not sensitive enough to distinguish different cognitive changes [54, 55], and even suggested that the Montreal Cognitive Assessment (MoCA) was a better choice for screening people with MCI [54]. The Hong Kong version of the MoCA was found to be reasonably good but not better than CMMSE in screening MCI [56]. Besides, using CMMSE as cognitive assessment would be easier for illiterate subjects since there was only one question related to verbal command. In addition, older illiterate respondents were only required to draw an overlapping pentagon in CMMSE, which was relatively easier than the clock drawing in the MoCA [56].

Table 3 General Estimating Equations (GEE) results for outcome variables

\begin{tabular}{|c|c|c|}
\hline Outcome Variables & B $(95 \% \mathrm{Cl})$ & $P$-value \\
\hline \multicolumn{3}{|l|}{ CMMSE } \\
\hline - Group & $0.17(-0.47,0.80)$ & 0.607 \\
\hline - Time & $0.10(-0.53,0.74)$ & 0.750 \\
\hline - Group* Time & $1.33(0.53,2.13)$ & $0.001^{*}$ \\
\hline \multicolumn{3}{|l|}{ IADL-CV } \\
\hline - Group & $0.51(-0.20,1.22)$ & 0.160 \\
\hline - Time & $-0.24(-0.85,0.38)$ & 0.445 \\
\hline - Group* Time & $1.07(0.34,1.81)$ & $0.004^{*}$ \\
\hline \multicolumn{3}{|c|}{$\begin{array}{l}{ }^{*} p \leq 0.05 \\
\text { T0, baseline of the study; T1, } 4 \text { months' post-intervention (T1 with the baseline } \\
\text { [T0] as reference). Only the model estimates of regression coefficients (B) of } \\
\text { the group (Group } 0=\text { control [reference], I= intervention). Effect sizes: Chinese } \\
\text { version of Mini-Mental State Examination (T1 vs T0), Cohen's } d=0.50 ; \text { Hong } \\
\text { Kong Chinese version of Lawton Instrumental Activities of Daily Living scale } \\
\text { (T1 vs T0), Cohen's } d=0.48 \\
\text { Abbreviations: } C I \text { confidence interval, CMMSE Chinese version of Mini-Mental } \\
\text { State Examination, IADL-CV Hong Kong Chinese version of Lawton Instrumen- } \\
\text { tal Activities of Daily Living scale }\end{array}$} \\
\hline
\end{tabular}

The results from this study confirmed the premise about the positive association between Tai Chi and global cognition in elderly persons with MCI. The findings supported previous studies of Tai Chi training in enhancing general cognitive functions of elderly persons with MCI [46, 57-60], as measured by MMSE. It was important to note that participants of the IG demonstrated significant improvement statistically after Tai Chi intervention on CMMSE and IADL-CV scores, when compared to the CG, but the scores changes did not exceed the limits of the respective MDCs in the current study. Such results indicated that the observed positive changes in CMMSE and IADL-CV scores were not large enough to rule out the possibility of measurement variation due to error. Larger scale studies are necessary to confirm the positive effects of Tai Chi on general cognition and IADL performance among elderly persons with MCI.

Tai Chi has been recognized as an alternate form of aerobic exercise, with a low-to-moderate intensity which is comparable to brisk walking [27]. In addition to aerobic efficacy, the meditation component of Tai Chi serves as a relaxation and stress reduction strategy, beneficent to preserve cognitive functions. As a cognition stimulating activity, practicing Tai Chi is found to improve attentional focus and executive function through learning series of movements in a continual sequence [30,31]. Therefore, Tai Chi training can be expected to give rise to more significant improvements in cognitive functions, as compared to other types of physical exercise. An earlier randomized control trial by Lam et al. $(2011,2012)$ compared the effectiveness of Tai Chi versus stretching and toning exercise in the maintenance of cognitive abilities in older Chinese people with MCI. Significant improvements in CMMSE and other cognitive assessments on memory and executions were found in both group at the five-month follow-up, but not at the one-year follow-up. Compared to the stretching and toning exercise group, there were fewer subjects from the Tai Chi group who had progressed to clinical dementia at five-month and one-year follow-up. 
However, the protective effect of Tai Chi in delaying dementia could not be confirmed because the Tai Chi group was better educated and had better baseline cognitive functions than the stretching and toning exercise group [46, 58]. In another randomized control trial by Mortimer et al. (2012), 120 non-demented older community-dwelling subjects were randomized to one of four groups, walking exercise, Tai Chi, social interaction, and no intervention (control). After a 40-week intervention, the Tai Chi group showed significant improvement in several neuropsychological measures including the Mattis Dementia Rating Scale, representing general cognitive functioning. The social interaction group also showed enhancements in some, but fewer, neuropsychological measures. No difference was noted for the walking exercise and control groups on any neuropsychiatric measure across time [61]. This finding suggested that Tai Chi was the best strategies for preserving cognitive functions in older adults. However, the result needed to be interpreted with caution because the sample population was subdivided into around 30 subjects participating in each study group. As the sample size of this study was too small, the possibility of a Type II error could not be overlooked. Moreover, this study did not obtain sufficient statistical power to detect significant differences between study groups for neuropsychiatric variables.

Although the present study supported positive effects of Tai Chi on general cognition of older adults with $\mathrm{MCI}$, there was no comparative exercise group, meaning that there was no provision to monitor the effects of Tai Chi relative to other types of physical exercise in enhancing general cognition of this population group.

There were vast differences in various cognitive studies in deciding the optimal dose and frequency of Tai Chi intervention. For the studies reported positive impacts on global cognitive functions, the durations of study interventions ranged from 14 [59] to 40 weeks [61] of regular instruction from a Tai Chi master. The frequencies of group sessions varied from once a week $[46,58]$ to three times a week $[60$, 61 and the session durations ranged from 40 [60] to $60 \mathrm{~min}$ [59]. In the present study, a 16-week short course of Tai Chi training was used, with participants meeting twice a week for 60 min per session, and this brought out positive changes in the global cognitive performances of this group of elderly persons with MCI. The implication of this clinical application can serve as a cost-effective cognitive health promotion program for the public.

Global cognitive status might be predictive of IADL performance in elderly persons [62, 63]. Functional evaluation of IADL performance has a central role in evaluating the progress of cognitive degeneration. However, there was limited empirical evidence to support the use of mind-body exercise interventions, to impact on
IADL performance in people suffering from MCI. After 16 weeks of Tai Chi training, the IG demonstrated a statistically-significant improvement in IADL performance, measured by the IADL-CV, compared to the usual-care CG who had not been assigned any physical activity. This is the first study indicating that Tai Chi can be considered as an effective strategy in modifying IADL performance for elderly persons with $\mathrm{MCI}$.

\section{Limitations}

Although the results seemed promising, the study was limited by not having adopted a randomized control trial, by using convenience sampling, and by not making any attempt to blind the investigator and participants in the study, leading to the risk of assessor and respondent biases, and by not making follow-up arrangements. In the current study, the recruitment process started after center assignment. Participants knew exactly what their treatment would be before they joined the study. It could enhance their willingness to participate [64] while it might have increased the likelihood of self-selection bias. Next, it remains unclear whether Tai Chi provides equal or superior cognitive benefits compared to other moderate intensity aerobic exercises. Future large-scale, well-conducted randomized control trials with appropriate comparison groups and follow-up arrangements are required to evaluate the long-lasting effect of Tai Chi on cognitive health.

\section{Conclusion}

Tai Chi is a mind-body therapy which comprises of both physical and intellectual components. It has been found to be a safe and culturally relevant exercise for elderly persons to practice. The results of this interventional study are promising, considering its short duration. The effects on general cognition and IADL performance in communitydwelling elderly persons with $\mathrm{MCI}$ are favorable.

\section{Additional file}

Additional file 1: Table S1. The Yang-style simple form of Tai Chi (DOCX $14 \mathrm{~kb}$ )

\section{Abbreviations \\ BADL: Basic activities of daily living: CG: Control group; Cl: Confidence interval; CMMSE: Chinese version of Mini-Mental State Examination; GEE: General Estimating Equations; IADL: Instrumental activities of daily living; IADL-CV: Hong Kong Chinese version of Lawton Instrumental Activities of Daily Living scale; IG: Intervention group; MCl: Mild cognitive impairment}

\section{Acknowledgments}

The authors sincerely thank all participants who joined the study, and $\mathrm{Mr}$ Cheung H. H., the Tai Chi master who provided the classes. Some special thanks are extended to Mr. Edgar Chow K. F. and Mr. Tang Y. C., who provided overall support for the recruitment and implementation processes of the trial.

Funding

No funding was applied for this study. 


\section{Availability of data and materials}

All data analyzed during this study are included in this published article and its Additional file.

\section{Authors' contributions}

MYS was responsible for development of study hypothesis, data analysis, and draft of the article. DTFL contributed to critical revision of the articles. All authors involved in the writing of this article, and approved the final submission.

\section{Ethics approval and consent to participate}

This study was performed in accordance with the Declaration of Helsinki and have been approved by the Joint CUHK-NTEC Clinical Research Ethics Committee of the Chinese University of Hong Kong (Clinical trial registry: CREC Ref. No.:2015.348). Retrospectively registered on ClinicalTrials.gov - NCT03404765. Written informed consent were signed by all participants before joining the study.

\section{Consent for publication}

Not Applicable. The manuscript does not contain any individual person's data.

\section{Competing interests}

The authors declare that they have no competing interest in this work.

\section{Publisher's Note}

Springer Nature remains neutral with regard to jurisdictional claims in published maps and institutional affiliations.

\section{Author details}

${ }^{1}$ The School of Nursing, Tung Wah College, 31 Wylie Road, Homantin, Kowloon, Hong Kong, SAR, People's Republic of China. ${ }^{2}$ The Nethersole School of Nursing, Chinese University of Hong Kong, Hong Kong, SAR, People's Republic of China.

Received: 27 June 2017 Accepted: 22 January 2018

Published online: 02 February 2018

\section{References}

1. World Health Organization. Dementia. Fact Sheet. Available from: http:// www.who.int/mediacentre/factsheets/fs362/en/ [Updated April 2016]. Accessed May 22, 2017.

2. Alzheimer's Disease International. Dementia in the Asia Pacific Region 2014. Available from: https://www.alz.co.uk/adi/pdf/Dementia-Asia-Pacific-2014. pdf. Accessed May 22, 2017.

3. Census and Statistics Department. Hong Kong Population Projections. 2015-2064. Available from: http://www.statistics.gov.hk/pub/ B1120015062015XXXXB0100.pdf. Accessed May 22, 2017.

4. $\quad$ LCW L, CWC T, WWC L, et al. prevalence of very mild and mild dementia in community-dwelling older Chinese people in Hong Kong. Int Psychogeriatr. 2008;20(1):135-48.

5. Yu R, Chau PH, McGhee SM, et al. Trends in prevalence and mortality of dementia in elderly Hong Kong population: projections, disease burden, and implications for long-term care. Int J Alzheimers Dis. 2012;2012:406852. https://doi.org/10.1155/2012/406852.

6. Petersen RC, Stevens JC, Ganguli M, Tangalos EG, Cummings JL, DeKosky ST. Practice parameter: early detection of dementia: mild cognitive impairment (an evidence-based review). Report of the quality standards Subcommittee of the American Academy of neurology. Neurology. 2001;56(9):1133-42.

7. Petersen $\mathrm{RC}$, Roberts $\mathrm{RO}$, Knopman DS, et al. prevalence of mild cognitive impairment is higher in men: the Mayo Clinic study of aging(CME). Neurology. 2010;75(10):889-97. https://doi.org/10.1212/WNL. 0b013e3181f11d85.

8. Pereira FS, Yassuda MS, Oliveira AM, Forlenza OV. Executive dysfunction correlates with impaired functional status in older adults. Int Psychogeriatr. 2008;20(6):1104-15. https://doi.org/10.1017/S1041610208007631.

9. Sánchez-Benavides G, Gómez-Ansón B, Quintana M, et al. Problem-solving abilities and frontal lobe cortical thickness in healthy aging and mild cognitive impairment. J Int Neuropsychol Soc. 2010;16(5):836-45. https://doi. org/10.1017/S135561771000069X.
10. Brown PJ, Devanand DP, Liu X, Caccappolo E. The Alzheimer's Disease Neuroimaging Initiative. Functional impairment in elderly patients with mild cognitive impairment and mild Alzheimer's disease. Arch General Psychiatry. 2011;68(6):617-26. https://doi.org/10.1001/archgenpsychiatry.2011.57.

11. Farias ST, Mungas D, Reed BR, Harvey D, Cahn-Weiner D, DeCarli C. MCl is associated with deficits in everyday functioning. Alzheimer Dis Assoc Disord. 2006;20(4):217-23. https://doi.org/10.1097/01.wad.0000213849.51495.d9.

12. Perneczky R, Pohl C, Sorg C, et al. Impairment of activities of daily living requiring memory or complex reasoning as part of the $\mathrm{MCl}$ syndrome. Int J Geriatr Psychiatry. 2006;21(2):158-62.

13. Reppermund S, Sachdev PS, Crawford J, et al. The relationship of neuropsychological function to instrumental activities of daily living in mild cognitive impairment. Int J Geriatr Psychiatry. 2011b;26(8):843-52. https:// doi.org/10.1002/gps.2612.

14. Tam CWC, Lam LCW, Chiu HFK, Lui WWC. Characteristics profiles of instrumental activities of daily living in Chinese older persons with mild cognitive impairment. Am J Alzheimers Dis Other Demen. 2007;22(3):211-7.

15. An GDA. Examination of instrumental activities of daily living assessment in older adults and mild cognitive impairment. J Clin Exp Neuropsychol. 2011; 34(1):11-34. https://doi.org/10.1080/13803395.2011.614598.

16. Luck T, Luppa M, Wiese $B$, et al. Prediction of incident dementia: impact of impairment in instrumental activities of daily living and mild cognitive impairment-results from the German study on ageing, cognition, and dementia in primary care patients. Am J Geriatr Psychiatry. 2012;20(11):94354. https://doi.org/10.1097/JGP.0b013e31825c09bc.

17. Purser $J$, Fillenbaum GG, Pieper CF, Wallace RB. Mild cognitive impairment and 10-year trajectories of disability in the lowa established populations for epidemiologic studies of the elderly cohort. J Am Geriatr Soc. 2005;53(11):1966-72.

18. Ganguli M, Snitz BE, Saxton JA, et al. outcomes of mild cognitive impairment depend on definition: a population study. Arch Neurol. 2011; 68(6):761-7. https://doi.org/10.1001/archneurol.2011.101.

19. Ritchie K, Artero S, Touchon J. Classification criteria for mild cognitive impairment: a population-based validation study. Neurology. 2001;56(1):37-42

20. Feldman $H$, Scheltens $P$, Scarpini $E$, et al. Behavioral symptoms in mild cognitive impairment. Neurology. 2004;62(7):1199-201.

21. Petersen RC, Thomas RG, Grundman M, et al. Vitamin E and donepezil for the treatment of mild cognitive impairment. N Engl J Med. 2005; 352:2379-88

22. Tricco AC, Soobiah C, Berliner S, et al. Efficacy and safety of cognitive enhancers for patients with mild cognitive impairment: a systematic review and meta-analysis. CMAJ. 2013;185(16):1393-401. https://doi.org/10.1503/ cmaj.130451.

23. Fratiglioni L, Paillard-Borg S, Winblad B. An active and socially integrated lifestyle in late life might protect against dementia. Lancet Neurol. 2004;3(6):343-53.

24. Verghese J, Lipton RB, Katz MJ, et al. Leisure activities and the risk of dementia in the elderly. N Engl J Med. 2003;348:2508-16.

25. Albaraly TN, Portet F, Fustinoni J, et al. Leisure activities and the risk of dementia in the elderly: results from the Three-City study. Neurology. 2009; 73(11):854-61. https://doi.org/10.1212/WNL.0b013e3181b7849b.

26. Li J, Hong Y, Chan K. Tai chi: physiological characteristics and beneficial effects on health. Br J Sports Med. 2001;35(3):148-56. https://doi.org/10. 1136/bjsm.35.3.148.

27. Taylor-Piliae RE. The effectiveness of tai chi exercise in improving aerobic capacity: an updated meta-analysis. Med Sport Sci. 2008;52:40-53. https:// doi.org/10.1159/000134283.

28. Colcombe SJ, Erickson Kl, Raz N, et al. aerobic fitness reduces brain tissue loss in aging humans. J Gerontol A Biol Sci Med Sci. 2003;58(2):176-80.

29. Colcombe SJ, Erickson Kl, Scalf PE, et al. Aerobic exercise training increases brain volume in aging humans. J Gerontol A Biol Sci Med Sci. 2006;61(11):1166-70.

30. Chiesa A, Calati R, Sterretti A. Does mindfulness training improve cognitive abilities? A systematic review of neuropsychological findings. Clin Psychol Rev. 2011;31(3):449-64. https://doi.org/10.1016/j.cpr.2010.11.003.

31. Kim THM, Pascual-Leone J, Johnson J, Tamim H. The mental-attention tai chi effect with older adults. BMC Psychol. 2016;4:29. https://doi.org/10.1186/ s40359-016-0137-0.

32. Cho KL. Effect of Tai Chi on depressive symptoms amongst Chinese older patients with major depression: the role of social support. 2008;52:146-54. https://doi.org/10.1159/000134295. 
33. Masi CM, Chen H-Y, Hawkley LC, Cacioppo JT. A meta-analysis of interventions to reduce loneliness. Personal Soc Psychol Rev. 2011;15(3): 219-66. https://doi.org/10.1177/1088868310377394.

34. Miller SM, Taylor-Piliae RE. Effects Of tai chi on cognitive function in community-dwelling older adults: a review. Geriatr Nurs. 2014;35(1):9-19. https://doi.org/10.1016/j.gerinurse.2013.

35. Wayne PM, Walsh JN, Taylor-Piliae RE, et al. the impact of tai chi on cognitive performance in older adults: a systematic review and meta-analysis. J Am Geriatr Soc. 2014;62(1):25-39. https:/doi.org/10.1111/jgs.12611.

36. Wu Y, Wang $Y$, Burgess EO, Wu J. The effects of tai chi exercise on cognitive function in older adults: a meta-analysis. J Sport Health Sci. 2013;2(4):193-203.

37. Zheng G, Liu F, Li S, Huang M, Tao J, Chen L. Tai chi and the protection of cognitive ability: A Systematic Review of Prospective Studies in Healthy Adults. Am J Prev Med. 2015;49(1):89-97. https://doi.org/10.1016/j.amepre. 2015.01.002

38. Pereira FS, Yassuda MS, Oliveira AM, et al. Profiles of functional deficits in mild cognitive impairment and dementia: benefits from objective measurement. J Int Neuropsychol Soc. 2010;16(2):297-305. https://doi.org/ $10.1017 /$ S1355617709991330

39. Dodge HH, Kadowaki T, Hayakawa T, Yamakawa M, Sekikawa A, Ueshima H. Cognitive impairment as a strong predictor of incident disability in specific ADL-IADL tasks among community-dwelling elders: the Azuchi study. Gerontologist. 2005;45(2):222-30. https://doi.org/10.1093/geront/45.2.222.

40. Millán-Calenti JC, Tubío J, Pita-Fernández S, Rochette S, Lorenzo T, Maseda A. Cognitive impairment as predictor of functional dependence in an elderly sample. Arch Gerontol Geriatr. 2012;54(1):197-201. https://doi.org/10. 1016/j.archger.2011.02.010.

41. Chiu HFK, Lee HC, Chung WS, Kwong PK. Reliability and validity of the Cantonese version of mini-mental state examination - a preliminary study. J Hong Kong College of Psychiatrists. 1994;4:25-8.

42. Chiu HF, Lam LC, Chi l, et al. Prevalence of dementia in Chinese elderly in Hong Kong. Neurology. 1998;50(4):1002-9.

43. Chibnall JT, Tait RC. Pain assessment in cognitively impaired and unimpaired older adults: a comparison of four scales. Pain. 2001;92(1-2):173-86.

44. Weiner D, Peterson B, Ladd K, McConnell E, Keefe F. Pain in nursing home residents: an exploration of prevalence, staff perspectives, and practical aspects of measurement. Clin J Pain. 1999;15(2):92-101.

45. Friedman TW, Yelland GW, Robinson SR. Subtle cognitive impairment in elders with mini-mental state examination scores within 'normal' range. Int J Geriatr Psychiatry. 2012;27(5):463-71. https://doi.org/10.1002/gps.2736.

46. Lam LC, Chau RC, Wong BM, et al. Interim follow-up of a randomized controlled trial comparing Chinese style mind body (tai chi) and stretching exercises on cognitive function in subjects at risk of progressive cognitive decline. Int J Geriatr Psychiatry. 2011;26(7):733-40. https://doi.org/10.1002/gps.2602.

47. Chow SC, Shao J, Wang H. Sample Size Calculation in clinical research. New York: Taylor and Frances Group; 2003.

48. Sannes TS, Mansky PJ, Chesney MA. The need for attention to dose in mind-body interventions: lessons from T'ai chi clinical trials. J Altern Complement Med. 2008;14(6):645-53. https://doi.org/10.1089/ acm.2007.0680.

49. Verhagen AP, Immink M, van der Meulen A, Bierma-Zeinstra SM. The efficacy of tai chi Chuan in older adults: a systematic review. Fam Pract. 2004;21(1):107-13.

50. Liang SY, Wu WC. Simplified Tai Chi Chuan 24 \& 48 Postures with Applications. 2nd ed. Roslindale: MA, YMAA Publishing Centre; 1996.

51. Folstein MF, Folstein SE, PR MH. Mini-mental state. A practical method for grading the cognitive state of patients for the clinician. J Psychiatr Res. 1975;12:189-98.

52. Tong YC, Man WK. The validation of the Hong Kong Chinese version of the Lawton instrumental activities of daily living scales for the institutionalized elderly persons. OTJR. 2002;22(4):132-42.

53. Lawton MP, Brody EM. Assessment of older people. Self-maintaining and instrumental activities of daily living. Gerontologist. 1969;9(3):179-86.

54. Lee JY, Lee DW, Cho SJ, et al. Brief screening for mild cognitive impairment in elderly outpatient clinic: validation of the Korean version of the Montreal cognitive assessment. J Geriatr Psychiatry Neurol. 2008;21(2):104-10. https:// doi.org/10.1177/0891988708316855.

55. Petersen RC, Smith GE, Waring SC, Ivnik RJ, Tangalos EG, Kokmen E. Mild cognitive impairment: clinical characterization and outcome. Arch Neurol. 1999:56(3):303-8.
56. Yeung PY, Wong LL, Chan CC, Leung JLM, Yun CC. A validation study of the Hong Kong version of Montreal Cognitive Assessment (HK-MoCA) in Chinese older adults in Hong Kong. Hong Kong Med J. 2014;20(6):504-10. https://doi.org/10.12809/hkmj144219.

57. Chang JY, Tsai P-F, Beck C, et al. the effect of tai chi on cognition in elders with cognitive impairment. Medsurg Nurs. 2011;20(2):63-70.

58. Lam LC, Chau RC, Wong BM, et al. A 1-year randomized controlled trial comparing mind body exercise (tai chi) with stretching and toning exercise on cognitive function in older Chinese adults at risk of cognitive decline. J Am Med Dir Assoc 2012;13(6):568.e15-568.568.e20. doi: https://doi.org/10. 1016/j.jamda.2012.03.008

59. Li F, Harmer P, Liu Y, Chou L-S. Tai Ji Quan and Global cognitive function in older adults with cognitive impairment: a pilot study. Arch Gerontol Geriatr. 2014;58(3):434-9. https://doi.org/10.1016/j.archger.2013.12.003.

60. Tsai P-F, Chang JY, Beck C, Kuo Y-F, Keefe FJ. A pilot cluster randomized trial of a 20-week tai chi program in elders with cognitive impairment and osteoarthritic knee: effects on pain and other health outcomes. J Pain Symptom Manag. 2013;45(4):660-9. https://doi.org/10.1016/j.jpainsymman. 2012.04.009.

61. Mortimer JA, Ding D, Borenstein AR, et al. Changes in brain volume and cognition in a randomized trial of exercise and social interaction in a community-based sample of non-demented Chinese elders. J Alzheimers Dis. 2012;30(4):757-66. https://doi.org/10.3233/JAD-2012-120079.

62. Aguero-Torres $H$, Thomas VS, Winblad B, Fratiglioni L. The impact of somatic and cognitive disorders on the functional status of the elderly. J Clin Epidemiol. 2002;55:1007-12.

63. McGuire LC, Ford ES, Ajani UA. Cognitive functioning as a predictor of functional disability in later life. Am J Geriatr Psychiatry. 2006;14(1):36-42.

64. Gross D, Fogg L. Clinical trials in the 21st century: the case for participantcentred research. Res Nurs Health. 2001;24(6):530-9.

\section{Submit your next manuscript to BioMed Central and we will help you at every step:}

- We accept pre-submission inquiries

- Our selector tool helps you to find the most relevant journal

- We provide round the clock customer support

- Convenient online submission

- Thorough peer review

- Inclusion in PubMed and all major indexing services

- Maximum visibility for your research

Submit your manuscript at www.biomedcentral.com/submit
C) Biomed Central 